\title{
PROGRAMA DE RESIDENTADO MÉDICO: PERCEPCIONES DE LOS MÉDICOS RESIDENTES EN HOSPITALES DE LIMA Y CALLAO
}

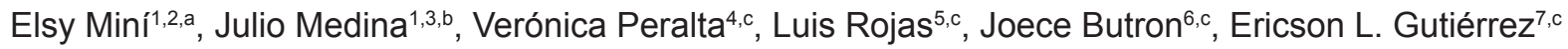

\begin{abstract}
RESUMEN
Con el objetivo de calificar la formación del programa de residentado médico desde las percepciones de los residentes, se aplicó una encuesta estructurada, basada en la literatura internacional a 228 participantes. El 48,2\% de los residentes calificó su formación como "buena", el 36,4\% como "regular" y 15,4\% como "mala". Los residentes, en su mayoría, tenían baja supervisión durante las guardias, se encontraban en sobrecarga laboral y no tenían descanso posguardia. Un buen plan de estudios anual (OR: 8,5; IC95\%: 4,1-7,4) y que la Universidad promueva trabajos de investigación (OR: 2,4; IC95\%: 1,1-5,2) fueron factores independientes asociados a una mejor calificación de la formación. En conclusión, la calificación de los residentes acerca de su formación en su mayoría es buena, pero este porcentaje no supera el $50 \%$, los entes formadores podrían utilizar estos resultados para proponer mejoras en los programas de formación de médicos residentes en el Perú.
\end{abstract}

Palabras clave: Formación de recursos humanos; Internado y residencia; Satisfacción laboral (fuente: DeCS BIREME).

\section{MEDICAL RESIDENCY PROGRAM: PERCEPTIONS OF MEDICAL RESIDENTS IN HOSPITALS OF LIMA AND CALLAO}

\begin{abstract}
In order to rate the medical residency training program from the perceptions of residents, a structured survey, based on international literature, was applied to 228 participants. $48.2 \%$ of residents rated their training as "good," $36.4 \%$ as "fair" and $15.4 \%$ as "poor". Most of the residents had low supervision while on call, were overworked and did not have rest after being on call. Having a good annual curriculum (OR: $8.5 ; 95 \% \mathrm{Cl}: 4.1$ to 7.4 ) and university promotion of research (OR 2.4, 95\% Cl: 1.1 to 5.2) were independent factors associated with higher ratings of training. In conclusion, the rating of residents about their training is mostly good, but this percentage does not exceed $50 \%$. Training authorities could use these results to propose improvements in training programs for medical residents in Peru.
\end{abstract}

Key words: Human resources formation; Internship and residency; Job Satisfaction (source: MeSH NLM).

\section{INTRODUCCIÓN}

La formación de segunda especialización en Medicina humana (Programa de Residentado Médico) comprende estudios escolarizados en sedes docentes (hospitales) con una duración de 3 a 5 años; en los cuales se debe cumplir anualmente 34 créditos de la práctica profesional en los hospitales y 6 créditos de 3 cursos de posgrado. El residente pasa, en su mayor tiempo, realizando su formación en la sede docente hospitalaria.
Las universidades y hospitales deben estar acreditadas por el Comité Nacional de Residentado Médico ${ }^{(1-3)}$.

En las sedes hospitalarias el residente realiza la práctica profesional dirigido por un coordinador de sede y supervisado por el tutor; estos profesionales médicos son acreditados y designados por la universidad. Además, se realizan actividades académicas y revisión de artículos científicos, que forman parte de su evaluación mensual o por rotación. En la universidad se

\footnotetext{
Instituto de Investigaciones Clínicas, Facultad de Medicina, Universidad Nacional Mayor de San Marcos. Lima, Perú.

Instituto Nacional Materno Perinatal. Lima, Perú.

Gerencia de Prestaciones Primarias de Salud, Gerencia Central, EsSALUD. Lima, Perú.

Hospital Sergio E. Bernales. Lima, Perú.

Oficina General de Investigación y Transferencia Tecnológica (OGITT). Instituto Nacional de Salud. Lima, Perú.

Oficina Ejecutiva de Planeamiento Estratégico. Hospital de Emergencias Pediátricas. Lima, Perú.

Facultad de Medicina, Universidad de San Martin de Porres. Lima, Perú.

a Médico cirujano, en especialidad en gineco-obstetricia, Mg en Salud Pública y Gestión Sanitaria. Doctor en Medicina (e), ${ }^{\mathrm{b}}$ médico cirujano con especialidad en pediatría, magíster Salud Pública (e), doctor en Medicina (e), ${ }^{\mathrm{c}}$ médico cirujano con especialidad en Gestión en Salud Recibido: : 25-09-14 Aprobado: 18-03-15
}

Citar como: Miní E, Medina J, Peralta V, Rojas L, Butron J, Gutiérrez EL. Programa de residentado médico: percepciones de los médicos residentes en hospitales de Lima y Callao. Rev Peru Med Exp Salud Publica. 2015;32(2):303-10. 
realiza, generalmente, el dictado de clases magistrales de los cursos programados en el Plan Anual. Cada universidad y sede hospitalaria tiene diferentes formas de docencia y evaluación del residente, material logístico e infraestructura diversa en la práctica formativa del residentado. Hace falta contar con un nivel formativo estandarizado ya que el Estado, mediante la Ley 28740 da importancia a la evaluación, acreditación y certificación de la calidad educativa en dichos centros ${ }^{(4)}$.

La educación médica tradicional no refleja la mejor evidencia de docencia, por tanto, las técnicas actuales utilizadas necesitan ser mejoradas e innovarse ${ }^{(5)}$. Hay autores que refieren que la perspectiva y opinión de los médicos residentes es uno de los más importantes recursos para mejorar la calidad del programa ofrecido ${ }^{(6-8)}$. La calidad educativa es determinada por la percepción desde la cual un programa de formación de residencia se ve, ya sea por los alumnos, los instructores, los reguladores, o de la sociedad ${ }^{(9)}$.

Las instituciones de salud exigen médicos especialistas adecuadamente capacitados, es por ello que los enfoques para asegurar la calidad en la educación médica deben seguir estándares óptimos ${ }^{(10,11)}$. Además, encuestas realizadas a los médicos residentes revelan que hay otros factores que contribuyen a una baja calidad del programa de residentado: el exceso de carga laboral, y el aumento de guardias y horas del jornal laboral, que generan fatiga y estrés en el residente ${ }^{(12,13)}$.

El estudio identifica una serie de factores asociados a la educación médica en el programa de residentado. Estos factores los hemos clasificado en aspectos de la entidad formadora (universidad) y la sede docente (hospital).

En el Perú es necesaria la formación de nuevos médicos especialistas con calidad académica e integral. El objetivo del estudio es calificar la formación recibida e identificar los factores relacionados en el residentado, desde la percepción de los médicos residentes de hospitales del Ministerio de Salud de Lima y Callao.

\section{EL ESTUDIO}

\section{DISEÑO Y POBLACIÓN DE ESTUDIO}

Se realizó un estudio analítico de corte transversal durante los meses de mayo a julio de 2012. Se incluyó en el estudio a médicos residentes de hospitales públicos del Ministerio de Salud de Lima y Callao, que estaban cursando del primero al tercer año de la residencia y que aceptaron participar en forma voluntaria. Se seleccionaron cinco hospitales de Lima y Callao, que contaron con la mayor cantidad de médicos residentes el 2012: Hospital Nacional Arzobispo Loayza, Hospital Nacional Dos de Mayo, Hospital Nacional Hipólito Unanue, Hospital Nacional María Auxiliadora y Hospital Nacional Daniel Alcides Carrión (Callao).

Para calcular el tamaño de la muestra se utilizó la fórmula para estimar proporciones en poblaciones finitas, considerando una prevalencia de 0,50, ya que no se conocía previamente el porcentaje de residentes con una formación calificada o medida como adecuada. Se estableció un nivel de confianza de 95\% y un error de $5 \%$. El número total de residentes de estos cinco hospitales fue de 560 , calculándose una muestra de 228 médicos residentes. Se estratificó la muestra de acuerdo a cada hospital, obteniéndose una muestra proporcional al número de residentes por hospital. La selección de los participantes del estudio fue no probabilístico por conveniencia, debido a la dificultad de encontrar a los residentes en las sedes docentes.

\section{VARIABLES}

Las variables independientes, generales de los residentes: sexo, edad, estado civil, año de graduación como médico cirujano, año de residencia, hospital, universidad, especialidad médica que realiza, tipo de plaza cautiva/libre (modalidad elegida al ingreso del residentado), guardias realizadas al mes, duración en horas de guardias y descanso posguardia. Además de la calificación del plan de estudios anual y la opinión de los residentes acerca de la promoción de trabajos de investigación por parte de la universidad.

Se construyó la variable dependiente (calificación de los médicos residentes acerca de su formación durante el residentado médico) mediante la integración de las siguientes variables hospitalarias y universitarias: desempeño del coordinador y del tutor, infraestructura y equipamiento hospitalario, supervisión durante las guardias e importancia de estas en la formación profesional, valoración de las clases magistrales, apoyo para la realización de proyectos de investigación, materiales y equipos universitarios, infraestructura y si las rotaciones se cumplen de acuerdo al perfil de la especialidad.

\section{PROCEDIMIENTOS}

Se elaboró un cuestionario basado en bibliografía internacional y nacional ${ }^{(14-16)}$ de 37 preguntas acerca de aspectos generales del residente, de la universidad y del hospital en relación a la formación del residentado médico, las preguntas fueron formuladas claramente y compuesta de 3 a 5 respuestas objetivas fue sometido a juicio de expertos por dos médicos con experiencia en investigación 
y diseño de encuestas en CAP (conocimientos, actitudes y prácticas). Se validó mediante una prueba piloto en 40 médicos residentes del Instituto Nacional Materno Perinatal, del Hospital Nacional Docente Madre Niño "San Bartolomé" y la Dirección de Salud Lima V Lima. Para evaluar la confiabilidad del instrumento se obtuvo un alfa de Cronbach de 0,9.

En la aplicación de la encuesta se explicó el objetivo del estudio, la forma de llenado y se obtuvo un consentimiento informado verbal. Las encuestas fueron llenadas por el residente en un lugar adecuado y luego recopiladas en sobre cerrado codificado, para ser enviadas al digitador. Finalizado el proceso de recolección de encuestas, se realizó el control de calidad de las mismas, se ordenó por código y sede hospitalaria y, por último, se hizo el llenado de la base de datos en el software respectivo.

\section{ANÁLISIS ESTADÍSTICO}

El análisis estadístico se realizó con el programa SPSS 18.0, se procedió al recopilado en la base de datos diseñada; para ello, cada pregunta del cuestionario fue codificada. Se realizó las pruebas de comprobación y consistencia de los datos, filtrando datos perdidos y omitidos. Se realizó el análisis descriptivo usando el porcentaje de frecuencias de las preguntas de las encuestas obtenidas.

Se obtuvieron tres tipos de resultados: el primero para obtener resultados descriptivos por pregunta, el segundo para un ranking de acuerdo a la formación del residentado y el tercero para analizar los factores asociados a la formación.

Para elaborar el ranking se realizó una sumatoria de las respuestas de las preguntas relacionadas a las variables acerca del hospital y universidad (muy buena, buena, regular, mala y muy mala) y se obtuvo la escala del ranking clasificada en "mala formación" (18 a 26 puntos), "regular formación" (27 a 32 puntos) y "buena formación" (33 a 44 puntos).

Para calcular el jornal laboral del médico residente, se adicionó preguntas acerca del número de guardias mensuales, la duración de las guardias y el horario de trabajo. Cada hospital lleva programado el tiempo y horario de guardias al mes y el residente conoce, al momento de la encuesta, esta información. Se consideró sobrecarga laboral si sobrepasaban 70 horas semanales, más de 10 guardias al mes y más de 12 horas continuas, según norma ${ }^{(1)}$.

Para el análisis bivariado se estudió la variable dependiente y las variables independientes mediante las pruebas de tendencia lineal y de chi cuadrado donde se estableció un nivel de significación de 0,05. Las variables que en el análisis bivariado tuvieron un valor de $p<0,05$ fueron introducidas en el modelo multivariado.

Lo médicos residentes participaron de forma voluntaria, previo consentimiento verbal; se aseguró el anonimato de los informantes; así mismo, los resultados fueron usados solo para fines del estudio. El estudio fue aprobado por el Instituto de Investigaciones Clínicas de la Facultad de Medicina de la Universidad Nacional Mayor de San Marcos, mediante comités de asesores en investigación y ética.

\section{HALLAZGOS}

Participaron 228 médicos residentes de cinco hospitales de Lima y Callao, el $67,0 \%$ de sexo masculino; la media de edad fue de $33 \pm 6,05$ años, el $50 \%$ entre 28 y 36 años de edad. El 39,9\% cursaban el primer año de residencia; el $25,9 \%$ laboraban en el Hospital Loayza y el $85,1 \%$ provenía de universidades nacionales. El $57,9 \%$ de especialidades clínicas y el $34,6 \%$ de quirúrgicas. El $21,9 \%$ de la especialidad de Medicina Interna, el $14,9 \%$ de Cirugía General y el 9,6\% de Ginecoobstetricia. Hubo una tasa de rechazo de menos del $1 \%$ (Tabla 1).

Tabla 1. Características generales de los médicos residentes hospitales de Lima y Callao 2012

\begin{tabular}{lcc}
\hline Características & $\mathbf{n}$ & $\mathbf{( \% )}$ \\
\hline Sexo & 152 & $(67,0)$ \\
$\quad$ Masculino & 76 & $(33,0)$ \\
\hline Femenino & & \\
Rango de edad & 155 & $(68,0)$ \\
\hline $25-34$ & 47 & $(20,6)$ \\
\hline $35-44$ & 14 & $(6,1)$ \\
\hline $45-54$ & 1 & $(0,4)$ \\
\hline $55-64$ & 11 & $(4,8)$ \\
\hline Datos en blanco & & \\
Año de residencia & 91 & $(39,9)$ \\
\hline Primero & 73 & $(32,0)$ \\
\hline Segundo & 64 & $(28,1)$ \\
\hline Tercer & & \\
Especialidad (por áreas) & 132 & $(57,9)$ \\
Clínicas & 79 & $(34,6)$ \\
\hline Quirúrgicas & 11 & $(4,8)$ \\
\hline Otras & 6 & $(2,6)$ \\
\hline Datos en blanco & & \\
Hospital & 59 & $(25,9)$ \\
\hline Hospital Arzobispo Loayza & 56 & $(24,6)$ \\
\hline Hospital Dos de Mayo & 42 & $(18,4)$ \\
\hline Hospital Hipólito Unanue & 41 & $(18,0)$ \\
\hline Hospital Daniel A Carrión & 30 & $(13,2)$ \\
\hline Hospital María Auxiliadora & & \\
\hline Tipo de universidad & 194 & $(85,1)$ \\
\hline Nacional & 34 & $(14,9)$ \\
\hline Particular & & \\
\hline & & \\
\hline
\end{tabular}


Tabla 2. Calificación de los médicos residentes acerca de los aspectos hospitalarios y universitarios que influyen en su formación

\begin{tabular}{lc}
\hline \multicolumn{1}{c}{ ¿Cómo calificaría el/la(s).......? } & $\begin{array}{c}\text { “Muy buena” } \mathbf{y} \\
\text { “buena" } \mathbf{n}(\%)^{*}\end{array}$ \\
\hline Hospital & $145(63,5)$ \\
\hline Calidad asistencial hospitalaria & $114(50,0)$ \\
\hline Actividades académicas hospitalarias & $111(48,6)$ \\
\hline Desempeño del tutor & $105(46,1)$ \\
Desempeño del coordinador & $55(24,1)$ \\
\hline Infraestructura hospitalaria & $51(22,4)$ \\
\hline Equipamiento tecnológico & \\
Universidad & $111(48,7)$ \\
\hline Plan de estudio anual & $104(45,6)$ \\
\hline Calidad de enseñanza universitaria & $81(35,5)$ \\
\hline Clases magistrales & $79(34,6)$ \\
Materiales y equipos & $69(30,3)$ \\
Infraestructura de la universidad & $51(22,4)$ \\
\hline Apoyo en trabajos de investigación & \\
\hline * muestra de 228 médicos residentes &
\end{tabular}

Los aspectos mejor valorados y calificados como "muy buena" y "buena" por los médicos residentes en el estudio fueron: la calidad asistencial hospitalaria (63,5\%); las actividades académicas (50\%); el plan de estudio anual $(48,7 \%)$ y la calidad de enseñanza universitaria $(45,6 \%)$ (Tabla 2).

El $86,0 \%$ contaba con tutor, sin embargo, el Hospital María Auxiliadora presentó un mayor porcentaje de residentes sin supervisión del tutor. El 23,7\% de los residentes refirieron de 10 a más guardias al mes; esto fue más frecuente en los hospitales Arzobispo Loayza, Hipólito Unanue, Daniel A. Carrión y María Auxiliadora. En promedio, los residentes realizaron guardias de $13 \mathrm{~h}$, y en los hospitales Arzobispo Loayza, Hipólito Unanue, Daniel A. Carrión y María Auxiliadora realizaron más de $12 \mathrm{~h}$ consecutivas de guardia (Tabla 3 ).

El $86,0 \%$ de los encuestados no cuentan con descanso posguardia y los residentes del Hipólito Unanue fueron los de mayor frecuencia. El 60,1\% refirió una supervisión

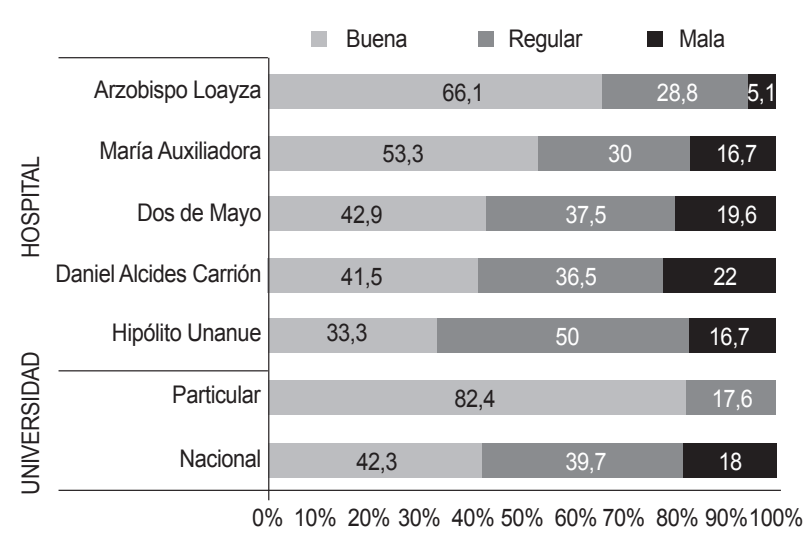

Figura 1. Formación recibida durante el residentado médico, según hospital y universidad de procedencia. Hospitales de Lima - Callao. 2012

en las guardias en menos del $50 \%$ de su labor diaria. La media de la jornada laboral fue de $74 \pm 17$ horas por semana. El $50 \%$ de los residentes se encontró en sobrecarga laboral y, de estos, la mayoría se encuentra en los hospitales Hipólito Unanue, Daniel A. Carrión y María Auxiliadora (Tabla 3).

Según el ranking elaborado, en general, el 48,2\% de los residentes calificó su formación como "buena", el $36,4 \%$ como "regular" y 15,4\% como "mala". El 66,1 y $53,3 \%$ de los residentes del Hospital Loayza y María Auxiliadora calificaron como "buena" la formación recibida respectivamente. El $82,4 \%$ de los residentes pertenecientes a las universidades particulares calificó igualmente como "buena" la formación recibida, sin embargo, en las universidades nacionales este porcentaje alcanzó un 42,3\% (Figura 1).

En el análisis bivariado, las variables asociadas a una mejor calificación de la formación recibida fueron: edad entre 31 a 57 años (OR: 1,8; IC95\%: 1,1-3,2, p=0,024); residentes de universidad particular (OR: 3,6; IC95\%: $0,9-13,1, \quad p<0,001)$; residentes de la especialidad

Tabla 3. Características del residentado médico en hospitales de Lima y Callao 2012

\begin{tabular}{|c|c|c|c|c|c|c|}
\hline \multirow{2}{*}{ Ítems/Hospital } & HNDM & HNAL & HNHU & HNDAC & HMA & Total \\
\hline & n (\%) & $\mathrm{n}(\%)$ & $\mathrm{n}(\%)$ & $\mathrm{n}(\%)$ & $\mathrm{n}(\%)$ & $\mathrm{n}(\%)$ \\
\hline Presencia del tutor & $47(83,9)$ & $55(93,2)$ & $35(83,3)$ & $32(78,0)$ & $27(90,0)$ & $196(86,0)$ \\
\hline No cuenta con supervisión del tutor * & $9(19,1)$ & $12(21,8)$ & $13(37,1)$ & $4(12,5)$ & $14(51,8)$ & $52(22,8)$ \\
\hline De 10 a más guardias al mes & $0(0,0)$ & $14(23,7)$ & $14(33,3)$ & $16(39,0)$ & $10(33,3)$ & $54(23,7)$ \\
\hline Duración de las guardias $>12$ horas & $0(0,0)$ & $14(23,7)$ & $9(21,4)$ & $4(9,8)$ & $9(30,0)$ & $192(84,2)$ \\
\hline No tiene descanso posguardia & $51(92,7)$ & $57(96,6)$ & $25(59,5)$ & $39(95,1)$ & $24(80,0)$ & $196(86,0)$ \\
\hline Guardias supervisadas < $50 \%$ & $30(53,6)$ & $29(49,1)$ & $35(83,3)$ & $26(63,4)$ & $17(56,6)$ & $137(60,1)$ \\
\hline Sobrecarga laboral & $5(9,8)$ & $21(35,6)$ & $31(73,8)$ & $29(70,7)$ & $28(93,3)$ & $114(50,0)$ \\
\hline
\end{tabular}

* Calculado de los que tienen tutor.

HNDM: Hospital Nacional Dos de Mayo; HNAL: Hospital Nacional Arzobispo Loayza; HNHU: Hospital Nacional Hipólito Unanue; HNDAC: Hospital Nacional Daniel Alcides Carrión, HMA; Hospital María Auxiliadora. 
quirúrgica (OR: 2; IC95\%: 0,9-3,6, p<0,016); un buen plan de estudios anual (OR: 9; IC95\%: 4,9-16,4, $p<0,001)$, y si la universidad promueve trabajos de investigación (OR: 2,6; IC95\%: 1,5-4,4, p<0,001).

Finalmente, en el análisis multivariado, el conjunto de variables que se encuentran asociadas a una mejor calificación acerca de la formación en el residentado son: un buen plan de estudios anual (OR: 8,5; IC95\%: 4,1-7,4, $p<0,001)$; a la promoción de trabajos de investigación (OR: 2,4; IC95\%: 1,1-5,2, $p=0,021$ ), y encontrarse haciendo la residencia en el Hospital Dos de Mayo (OR: 5,8; IC95\%: 1,8-18,3, $p=0,003)$; en el Hospital Arzobispo Loayza (OR: 4,5; IC95\%: 1,4-14,5), y en el Hospital Daniel Alcides Carrión (OR: 3,7; IC95\%: 1,2-12,1) (Tabla 4).

Tabla 4. Factores asociados a la formación de los médicos residentes en hospitales de Lima y Callao, 2012

\begin{tabular}{|c|c|c|c|c|c|}
\hline \multirow[b]{2}{*}{ Variables } & \multirow[b]{2}{*}{$\mathbf{N}(\%)$} & \multicolumn{2}{|c|}{ Modelo crudo } & \multicolumn{2}{|c|}{ Modelo ajustado } \\
\hline & & OR (IC 95\%) & $\begin{array}{c}\text { valor } \\
p\end{array}$ & OR (IC 95\%) & $\begin{array}{c}\text { valor } \\
p\end{array}$ \\
\hline \multicolumn{6}{|l|}{ Mujer } \\
\hline No & $71(46,7)$ & 1,0 & & & \\
\hline Sí & $39(51,3)$ & $1,2(0,7-2,1)$ & 0,512 & & \\
\hline \multicolumn{6}{|c|}{ Rango de edad } \\
\hline 23 a 30 años & $48(40,7)$ & 1,0 & & & \\
\hline 31 a 57 años & $57(55,9)$ & $1,8(1,1-3,2)$ & 0,024 & $1,6(0,8-3,1)$ & 0,210 \\
\hline \multicolumn{6}{|l|}{ Estado civil } \\
\hline Unido & $41(48,8)$ & 1,0 & & & \\
\hline Soltero & $69(47,9)$ & $1(0,6-1,7)$ & 0,896 & & \\
\hline \multicolumn{6}{|c|}{ Año de residencia } \\
\hline 3. ${ }^{\text {er }}$ Año & $38(59,4)$ & - & 0,104 & & \\
\hline 2. ${ }^{\circ}$ Año & $31(42,5)$ & - & & & \\
\hline 1. er Año & $41(45,1)$ & - & & & \\
\hline \multicolumn{6}{|c|}{ Sede hospitalaria } \\
\hline HNHU & $14(33,3)$ & - & 0,011 & 1,0 & \\
\hline HNAL & $39(66.1)$ & - & & $4,5(1,4-14,5)$ & 0,011 \\
\hline HNMA & $16(53,3)$ & - & & $3,2(0,9-11,8)$ & 0,082 \\
\hline HNDM & $24(42,9)$ & - & & $5,8(1,9-18,3)$ & 0,003 \\
\hline HNDAC & $17(41,5)$ & - & & $3,7(1,2-12,1)$ & 0,027 \\
\hline \multicolumn{6}{|c|}{ Sobrecarga laboral } \\
\hline No & $57(50,0)$ & 1,0 & & & \\
\hline Sí & $53(46,5)$ & $1,2(0,7-1,9)$ & 0,596 & & \\
\hline \multicolumn{6}{|c|}{ Universidad particular } \\
\hline No & $82(42,3)$ & 1,0 & & & \\
\hline Sí & $28(82,4)$ & $6,4(2,5-16,1)$ & $<0,001$ & $3,6(0,9-13,1)$ & 0,056 \\
\hline \multicolumn{6}{|c|}{ Especialidad quirúrgica } \\
\hline No & $61(42,7)$ & 1,0 & & & \\
\hline Sí & $47(59,5)$ & $2,0(1,1-3,5)$ & 0,016 & $1,8[0,9-3,6]$ & 0,110 \\
\hline \multicolumn{6}{|c|}{ Buen plan de estudios } \\
\hline No & $28(23,9)$ & 1,0 & & & \\
\hline Sí & $82(73,9)$ & $9(4,9-16,4)$ & $<0,001$ & $8,5(4,1-7,4)$ & $<0,001$ \\
\hline \multicolumn{6}{|c|}{ Promueve trabajos de investigación } \\
\hline No & $40(36,4)$ & 1,0 & & & \\
\hline Sí & $70(59,3)$ & $2,6(1,5-4,4)$ & 0,001 & $2,4(1,1-5,2)$ & 0,021 \\
\hline
\end{tabular}

\section{DISCUSIÓN}

El presente estudio encuentra que el $48,2 \%$ de los residentes calificó su formación como "buena", el 36,4 \% como "regular" y $15,4 \%$ como "mala". Un estudio previo realizado en residentes de Psiquiatría de Lima refiere que el $37,8 \%$ de ellos consideró estar satisfecho con su formación ${ }^{(17)}$. Otro estudio reciente realizado en 24 residentes de Lima, demostró que 20 de ellos (87\%) calificaron su formación como buena o muy buena (18). Las diferencias con estos estudios se deberían a que en los estudios mencionados se evalúa la satisfacción global con una sola pregunta en escala de Likert, a diferencia del presente estudio que evalúa varios aspectos, tanto hospitalarios como universitarios de la formación del residente.

Un porcentaje mayor de residentes satisfechos con su formación se encontró en un estudio realizado en España (87\%), así mismo, en Paraguay el porcentaje de residentes que calificaron su formación como buena y muy buena llego al $60 \%(19,20)$.

Se encontró que la edad promedio de residentes fue similar a otros estudios realizados (14,21); sin embargo, la mayoría de participantes fueron de sexo masculino a diferencia de otros estudios realizados en España ${ }^{(22,23)}$. En investigaciones de Barcelona se ha asociado al ambiente donde se desarrolla el residente al logro de los objetivos de la residencia y, por ende, en su formación. En el presente estudio, la calificación de la infraestructura y equipamiento hospitalario es desfavorable y se refleja en una calificación de la formación menos valorada ${ }^{(24)}$.

Las actividades académicas y asistenciales realizadas en las sedes hospitalarias son importantes porque refuerzan la formación del residente, en el presente estudio obtuvo en general una buena calificación. En otros estudios hasta un $70 \%$ de los residentes califican de igual forma estas actividades que contribuyen a desarrollar habilidades clínicas y académicas del residente ${ }^{(22,25)}$.

El desempeño del personal a cargo de la formación de los residentes es fundamental para incrementar la calidad educativa, además que es un articulador entre el hospital y la universidad. Se encontró una baja calificación en más de la mitad de los residentes encuestados con respecto al desempeño del coordinador y tutor. Estos últimos, según estudios, deben poseer una sólida instrucción en contenidos metodológicos y herramientas que faciliten la labor docente ${ }^{(26)}$. Los médicos residentes que calificaron mejor la formación recibida, valoraron mejor a sus tutores; en esta investigación se encontró esta relación, tan igual como en otros estudios ${ }^{(6,14,27)}$. 
La mayoría de residentes contaban con tutor y el $22,8 \%$ no fueron supervisados por este, o fueron supervisados por otros profesionales no acreditados. La presencia del tutor es fundamental en esta labor, sin embargo, estudios en EE. UU. y Nigeria reflejan que, igualmente, hay una disminución de la supervisión por parte del personal encargado ${ }^{(15,21)}$. Por otro lado, este hallazgo puede variar y depende de la especialidad, donde los porcentajes de supervisión pueden superar hasta el 90\%, como ocurre en especialidades quirúrgicas en España ${ }^{(28)}$.

Según los médicos encuestados, en los hospitales Arzobispo Loayza, Daniel Alcides Carrión, Hipólito Unánue y Maria Auxiliadora los residentes superaron las diez guardias al mes, de más de $12 \mathrm{~h}$ de duración y sin descanso posguardia, lo que incumple la normativa sobre el residentado médico en el país ${ }^{(1)}$. Se encontró en países europeos que las guardias, su duración y jornal laboral están controlados; además, refieren que las horas fuera del horario de trabajo, que el residente dedica al estudio personal, son absolutamente claves en la calidad de formación que el residente va adquirir ${ }^{(21,28,29)}$.

Se evidenció la existencia de sobrecarga laboral, principalmente en los hospitales Daniel Alcides Carrión, Hipólito Unanue y María Auxiliadora. En diversos países se encuentra un jornal laboral de $40 \mathrm{~h}$ por semana, cuyo valor es muy inferior al encontrado en nuestro país ${ }^{(22,29)}$. Hay estudios que evidencian que la sobrecarga laboral genera fatiga, aumento de sueño, estrés, falta de motivación para realizar su labor y preparar sus actividades académicas; por lo que es necesario tomar medidas para el cumplimiento de las horas según normativa en nuestro país ${ }^{(30,31)}$. En EE. UU. no se ha llegado a un consenso como afectaría a la calidad educativa, sin embargo, dichos estudios concuerdan que un médico residente con sobrecarga laboral tiene mayores errores médicos ${ }^{(10,12)}$.

En las universidades, una de las actividades menos valoradas por los residentes fueron las clases magistrales. Estos hallazgos están en relación con otros estudios donde se observa que el residente en esta modalidad tiene un papel pasivo en su formación, además de una baja capacidad de retener e influir en la valoración de su formación ${ }^{(6,31)}$. De diversas metodologías usadas, como el aprendizaje basado en problemas o las demostraciones prácticas, las enseñanzas tradicionales en el aula tipo conferencia o magistrales no dan los mejores resultados, sin embargo, en nuestro país se continúa usando esta metodología de enseñanza ${ }^{(32)}$.

Con respecto a la investigación, la mayoría de participantes opinó que hay falta de apoyo o estímulo por parte de la universidad para la realización de trabajos de investigación, esto es más preocupante en universidades nacionales; estos resultados son similares a otros estudios ${ }^{(14,33)}$. Los aspectos relacionados a incrementar la investigación durante el residentado es fundamental para la formación del médico y se puede realizar de diversas maneras, una de estas es involucrándolo en las actividades asistenciales y académicas ${ }^{(26,34)}$.

Los factores que influyeron positivamente en la calificación por el residente, como el rango de edad entre 31 a 57 años, la universidad y hospital de procedencia fueron relacionados en el modelo bivariado, sin embargo, en el modelo ajustado, las variables: un buen plan de estudios anual y la promoción de trabajos de investigación fueron asociadas a una buena formación, diferente a otros estudios ${ }^{(14,22,27)}$.

Una de las limitaciones de la presente investigación es que los resultados solo fueron obtenidos en residentes de hospitales del Ministerio de Salud, sin embargo, un grupo importante de residentes se encuentran en hospitales del seguro social y fuerzas armadas, los cuales no fueron incluidos en el estudio por las limitaciones en la coordinación con estas sedes. Sin embargo, estos resultados pueden ser extrapolables a estas poblaciones, ya que las condiciones hospitalarias y universitarias del residentado son similares.

En conclusión, la calificación de los residentes acerca de su formación en su mayoría fue buena, pero este porcentaje no supera el $50 \%$. El plan de estudios, el apoyo a la investigación, el cambio de metodología usada en las clases, la presencia de un tutor calificado que acompañe y/o supervise las actividades de los residentes, son entre otros aspectos, los que se podrían mejorar para contribuir a la mejora de la calidad de la educación médica durante el proceso de formación de médicos residentes en el Perú.

Contribuciones de autoría: EM, LR y VP Concepción y diseño del artículo. EM y JM Análisis e interpretación de datos. Redacción del artículo. Revisión crítica del artículo. EM, JM, LR, VP Aprobación de la versión final. Asesoría técnica y administrativa. EM Obtención de financiamiento. LR, VP, EG, JB Recolección de resultados. Análisis e interpretación de datos. Redacción del artículo. Revisión crítica del artículo. Asesoría estadística. Asesoría técnica o administrativa.

Conflictos de interés: Joece Butron, Ericson Gutiérrez, Verónica Peralta y Luis Rojas, realizaron el programa de residentado médico por la Universidad Nacional Mayor de San Marcos durante la elaboración de esta investigación.

Fuentes de financiamiento: Consejo Superior de Investigación - Vicerrectorado Académico UNMSM, Código Concytec: 02020002, Código UNESCO: 320199. 


\section{REFERENCIAS BIBLIOGRÁFICAS}

1. Comité Nacional de Residentado Médico. . Reglamento del Sistema Nacional del Residentado Médico. Resolución Suprema N002-2006SA. Lima: CONAREME; 2006.

2. Comité Nacional de Residentado Médico. Normas Básicas del Sistema Nacional del Residentado Médico. Decreto Supremo No 008-88-SA. Lima: CONAREME; 1988.

3. Burstein Alva Z. Historia de los programas de formación y titulación de médicos especialistas en el Perú. Rev Peru Med Exp Salud Publica. 2014;31(3):598-600.

4. Perú, Congreso de la República. Ley del Sistema Nacional de Evaluación, Acreditación y Certificación de la Calidad Educativa. Ley No28740. El Peruano. 23 may 2006.

5. Satterlee WG, Eggers RG, Grimes DA. Effective medical education: insights from the Cochrane Library. Obstet Gynecol Surv. 2008 May;63(5):32933.

6. Jalili M, Mirzazadeh A, Azarpira A. A survey of medical students' perceptions of the quality of their medical education upon graduation. Ann Acad Med Singapore. 2008 Dec;37(12):1012-8.

7. Neacy K, Stern SA, Kim HM, Dronen SC. Resident perception of academic skills training and impact on academic career choice. Acad Emerg Med. 2000 Dec;7(12):1408-15.

8. Kozakowski SM, Wieschhaus MF, Abercrombie S, Carr S, Gravel J, Hall KL, et al. Quality in residency education. Ann Fam Med. 2010;8(3):271.

9. Sisson SD, Casagrande SS, Dalal D, Yeh H-C, Johns Hopkins University School of Medicine. Associations between quality indicators of internal medicine residency training programs. BMC Med Educ. 2011;11:30,

10. Fitzgibbons SC, Chen J, Jagsi R, Weinstein D. Long-term followup on the educational impact of ACGME duty hour limits: a prepost survey study. Ann Surg. 2012 Dec;256(6):1108-12. doi: 10.1097/ SLA.0b013e31825ffb33.

11. Reiter ER, Wong DR. Impact of duty hour limits on resident training in otolaryngology. Laryngoscope. 2005
May;115(5):773-9.

12. Noroozi A-R, Philbert RF. Residents' perception of the impact of the Bell Commission in oral surgery training: an initial study. J Oral Maxillofac Surg. 2008 Jul;66(7):1329-34. doi: 10.1016/j.joms.2008.01.044.

13. Borman KR, Jones AT, Shea JA. Duty hours, quality of care, and patient safety: general surgery resident perceptions. J Am Coll Surg. 2012 Jul;215(1):70-7; discussion 77-9. doi: 10.1016/j. jamcollsurg.2012.02.010.

14. González-Martínez JF, García-García JA, Del Rosario Arnaud-Viñas M, Arámbula-Morales EG, UriegaGonzález Plata S, Mendoza-Guerrero JA. Assessment of medical residents' satisfaction. Cir Cir. 2011 MarApr;79(2):156-67.

15. Baldwin DWC, Daugherty SR, Ryan PM. How Residents View Their Clinical Supervision: A Reanalysis of Classic National Survey Data. J Grad Med Educ. 2010 Mar;2(1):37-45.

16. Santana Cabrera L, Sánchez-Palacios M, Rodríguez González F, Hernández Medina E, Casamitjana Ortega A, Fernández Arroyo M. Actitudes y percepciones del personal médico del hospital acerca de los Cuidados Intensivos y de la especialidad de Medicina Intensiva. Med Intensiva. 2008;32(7):319-28

17. Ocampo-Zegarra JC, Cortez-Vergara C, Alva-Huerta M, Rojas-Rojas G. Encuesta a médicos residentes de psiquiatría sobre la calidad de su formación como especialistas. Rev Neuropsiquiatr. 2014;76(2):109.

18. Herrera-Añazco P, Hernández AV, Sánchez-Rivas F, Arana Maestre C. Percepción de la formación durante el residentado médico en hospitales del Ministerio de Salud de Lima, Perú. Rev Peru Med Exp Salud Publica. 2014;31(3):606-7.

19. Moro J, Tejedor JM, Zancajo JL. La calidad de la formación especializada a través de la encuesta de opinión de residentes. Rev Calid Asist. 2006;21(2):82-6.

20. Ortiz DD, Vidovich LF, Noveri JLM, Canata ME de. Percepción de Médicos Residentes sobre la Calidad de sus Programas de Formación. Rev Sal Publica Parag. 2014;4(2):16-25.
21. Yusufu LM, Ahmed A, Odigie VI, Delia IZ, Mohammed AA. Residency training program: perceptions of residents. Ann Afr Med. 2010 AprJun;9(2):91-4. doi: 10.4103/15963519.64745 .

22. Ayala-Morillas LE, Fuentes-Ferrer ME, Sánchez-Díaz J, Rumayor-Zarzuelo M, Fernández-Pérez C, Marco-Martínez F. Factors associated with residents satisfaction with their training as specialists. Rev Clin Esp (Barc). 2014 May;214(4):175-83.

23. Meijide-Míguez H, Rey RR, Martínez F de la I. La formación de Médicos Internos Residentes de Medicina Interna en Galicia: encuesta de opinión. Galicia Clin. 2010;71(4):153-9.

24. Oristrell J, Oliva JC, Casanovas A, Comet R, Jordana R, Navarro M. The Computer Book of the Internal Medicine Resident: competence acquisition and achievement of learning objectives. Rev Clin Esp (Barc). 2014 Jan-Feb;214(1):8-16. doi: 10.1016/j. rce.2013.07.010.

25. Ackerly DC, Sangvai DG, Udayakumar K, Shah BR, Kalman NS, Cho AH, et al. Training the next generation of physician-executives: an innovative residency pathway in management and leadership. Acad Med. 2011 May;86(5):575-9. doi: 10.1097/ ACM.0b013e318212e51b.

26. Sánchez Marín FJ, Molina Durán F, Romero Sánchez BE. Capacidad y necesidades del residente. Recursos docentes para ayudar al tutor en una situación de cambio. Aten Primaria. 2007 Oct;39(10):535-9.

27. Pérez-Romero FJ, Chacón Caso $P$, Casado Vicente V, Cerezuela FP. Percepción de los Residentes de Medicina Familiar y Comunitaria sobre el programa formativo de la Especialidad. Rev Clin Med Fam. 2012;5(1):17-24

28. Miguelena Bobadilla JM, Morales García D, Serra Aracil X, Sanz Sánchez M, Iturburu I, Docobo Durántez F, et al. [Training of residents in abdominal wall surgery in Spain]. Cir Esp. 2013 Feb;91(2):72-7. doi: 10.1016/j. ciresp.2011.08.004. [Artículo en Español].

29. Struhal W, Sellner J, Lisnic V, Vécsei L, Müller E, Grisold W. Neurology residency training in Europe - the 
current situation. Eur J Neurol. 2011 Apr;18(4):e36-40. doi: 10.1111/j.1468-1331.2010.03219.x.

30. Schwartz A, Pappas C, Bashook PG, Bordage G, Edison M, Prasad B, et al. Conceptual frameworks in the study of duty hours changes in graduate medical education: a review. Acad Med. 2011 Jan;86(1):18-29. doi: 10.1097/ ACM.0b013e3181ff81dd.

31. Alguersuari Cabiscol A, Borrat Padrós A, Del Cura Rodrguez JL. El papel del residente en su formación y en la actividad docente del servicio de radiodiagnóstico. Radiologia. 2010 Sep-Oct;52(5):456-60. doi: 10.1016/j. rx.2010.05.004.

32. García-Jiménez EP, Rojas-Pérez EM, Ruiz-Ruisánchez A. Técnicas de estudio para mejorar el aprendizaje en la residencia médica. Rev Mex Anest. 2012;35(S1):S242-4.

33. Vázquez IÍ, Bran DR, Fernández MM, Valcarcel PV, Rey RR, Monte R. Actividad investigadora de los residentes de medicina interna de Galicia. Galicia Clin 2012;73(1):710.
34. Figueroa L. ¿Existe un espacio para la investigación durante el residentado médico en Perú?. Rev Peru Med Exp Salud Pública. 2011;28(4):692-3.

Correspondencia: Elsy Haydeé Miní Diaz Dirección: Calle San Marcos D5 Urbanización El Remanso, Lima 3, Perú. Teléfono: (511) 3563391 - 999493820 Correo electrónico:elsyminidiaz@hotmail.com

\section{Consulte la versión electrónica de la Revista Peruana de Medicina Experimental y Salud Pública en} www.pubmed.gov

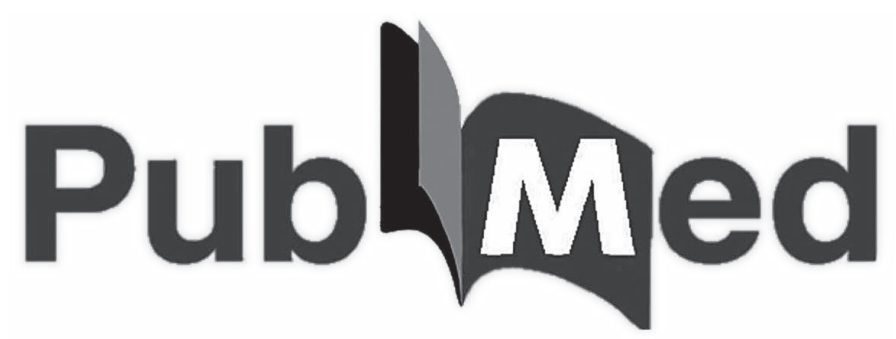

